\title{
The V1647 Orionis (IRAS 05436-0007) Protostar and Its Environment
}

\section{Citation}

McGehee, Peregrine M., J. Allyn Smith, Arne A. Henden, Michael W. Richmond, Gillian R. Knapp, Douglas P. Finkbeiner, Željko Ivezić, and J. Brinkmann. 2004. "The V1647 Orionis (IRAS 054360007) Protostar and Its Environment." The Astrophysical Journal 616 (2) (December): 1058-1064. doi:10.1086/425069.

\section{Published Version}

doi:10.1086/425069

\section{Permanent link}

http://nrs.harvard.edu/urn-3:HUL.InstRepos:33462005

\section{Terms of Use}

This article was downloaded from Harvard University's DASH repository, and is made available under the terms and conditions applicable to Other Posted Material, as set forth at http:// nrs.harvard.edu/urn-3:HUL.InstRepos:dash.current.terms-of-use\#LAA

\section{Share Your Story}

The Harvard community has made this article openly available.

Please share how this access benefits you. Submit a story.

\section{Accessibility}




\title{
THE V1647 ORIONIS (IRAS 05436-0007) PROTOSTAR AND ITS ENVIRONMENT
}

\author{
Peregrine M. McGehee, ${ }^{1,2}$ J. Allyn Smith, ${ }^{3,4}$ Arne A. Henden, ${ }^{5}$ Michael W. Richmond, ${ }^{6}$ Gillian R. Knapp, ${ }^{7}$ \\ Douglas P. Finkbeiner, ${ }^{7}$ Željko Ivezić, $^{7}$ and J. Brinkmann ${ }^{8}$ \\ Received 2004 July 5; accepted 2004 August 11
}

\begin{abstract}
We present Sloan Digital Sky Survey (SDSS) and United States Naval Observatory (USNO) observations of the V1647 Ori protostar and its surrounding field near NGC 2068. V1647 Ori, the likely driving source for HH 23, brightened significantly in 2003 November. Analysis of SDSS imaging acquired in 1998 November and 2002 February during the quiescent state, recent USNO photometry, and published Two Micron All Sky Survey (2MASS) and Gemini data show that the color changes associated with brightening suggest an EX Lupi type (EXor) outburst rather than a simple dust-clearing event.
\end{abstract}

Subject headings: circumstellar matter — stars: individual (IRAS 05436-0007, V1647 Orionis) stars: formation — stars: pre-main-sequence

Online material: color figure

\section{INTRODUCTION}

In 2004 January, J. W. McNeil discovered a new reflection nebula in the dark cloud Lynds 1630 near M78 (McNeil 2004). This object, now known as McNeil's Nebula, is apparently associated with an EX Lupi type (EXor-type) eruption (Reipurth \& Aspin 2004) of the embedded protostar V1647 Ori.

EX Lupi type stars (EXors) belong to the class of pre-mainsequence optical variables (Herbig 1977). They are classical $\mathrm{T}$ Tauri stars that undergo irregular optical/UV outbursts of several magnitudes, named for the prototype EX Lup (Herbig et al. 2001). These outbursts are interpreted as episodes of substantial mass transfer resulting from instabilities in the accretion disk; they are present very early in the evolution of a protostar, as shown by the detection of EXor outbursts from deeply embedded Class I protostars in the Serpens star formation region (Hodapp et al. 1996).

Clark (1991) first identified V1647 Ori as the young stellar object IRAS $05436-0007$ on the basis of its IRAS colors. $I$-band and [S II] narrow-band imaging of the region by Eislöffel $\&$ Mundt (1997) revealed a faint $I$-band source at the position of the IRAS object and reflection nebulosity extending to the north, identifying V1647 Ori as the likely driver for $\mathrm{HH} 23$, located $170^{\prime \prime}$ north of the star. The bolometric flux of the source derived from IRAS and submillimeter photometry by Lis et al. (1999) yields a luminosity of $2.7 L_{\odot}$ and an inferred molecular gas mass of $0.4 M_{\odot}$, assuming a distance of $400 \mathrm{pc}$ to the Orion star-forming complex (Anthony-Twarog 1982).

\footnotetext{
1 Los Alamos National Laboratory, LANSCE-8, MS H820, Los Alamos, NM 87545

2 Department of Astronomy, New Mexico State University, MSC 4500, Box 30001, Las Cruces, NM 88003; peregrin@apo.nmsu.edu.

3 Los Alamos National Laboratory, ISR-4, MS D448, Los Alamos, NM 87545 .

4 Department of Physics and Astronomy, University of Wyoming, 1000 East University Boulevard, Laramie, WY 82071.

5 Universities Space Research Association and US Naval Observatory, Flagstaff Station, P.O. Box 1149, Flagstaff, AZ 86002.

6 Department of Physics, Rochester Institute of Technology, 85 Lomb Memorial Drive, Rochester, NY 14623.

7 Princeton University Observatory, Princeton, NJ 08544.

8 Apache Point Observatory, 2001 Apache Point Road, Sunspot, NM 88349.
}

Estimates of the extinction toward V1647 Ori, $A_{V}=11-$ $15 \mathrm{mag}$, are found to be similar from photometry taken during the quiescent phase (Abraham et al. 2004) and during the eruptive phase (Reipurth \& Aspin 2004; Vacca et al. 2004; Briceño et al. 2004; Andrews et al. 2004). Thus, it is not clear whether the appearance of McNeil's Nebula is due to only the eruption of V1647 Ori or to the eruption plus additional clearing of obscuring circumstellar dust. In this paper we examine this question using preeruption multiband optical and near-IR data from the Sloan Digital Sky Survey (SDSS) and the Two Micron All Sky Survey (2MASS) compared with posteruption data in the SDSS and 2MASS bands observed at the United States Naval Observatory (USNO).

\section{OBSERVATIONS}

We detect the protostar at four epochs of SDSS imaging as a point source (SDSS J054613.14-000604.1), coincident with the 2MASS $K$-band position $\left(\alpha_{2000}=05^{\mathrm{h}} 46^{\mathrm{m}} 13.1, \delta_{2000}=\right.$ $\left.-00^{\circ} 06^{\prime} 05^{\prime \prime}\right)$. The SDSS observations consist of two pairs of scans acquired in 1998 November and 2002 February. Figure 1 shows an SDSS composite image, made with the riz filters, in which the protostar and the faint nebulosity to the north can be seen.

A technical summary of the SDSS is given by York et al. (2000). The SDSS imaging camera is described by Gunn et al. (1998). The Early Data Release and the Data Release One are described by Stoughton et al. (2002) and Abazajian et al. (2003). The former includes an extensive discussion of the data outputs and software. Pier et al. (2003) describe the astrometric calibration of the survey, and the network of primary photometric standard stars is described by Smith et al. (2002). The photometric system itself is defined by Fukugita et al. (1996), and the system that monitors the site photometricity is defined by Hogg et al. (2001). Abazajian et al. (2003) discuss the differences between the native SDSS $2.5 \mathrm{~m}$ ugriz system and the $u^{\prime} g^{\prime} r^{\prime} i^{\prime} z^{\prime}$ standard star system defined on the USNO $1.0 \mathrm{~m}$ (Smith et al. 2002). The SDSS low Galactic latitude data, which includes the Orion equatorial imaging used in this work, are described by Finkbeiner et al. (2004a).

The USNO Flagstaff station $1.0 \mathrm{~m}$ and $1.55 \mathrm{~m}$ telescopes were used to obtain eruptive-phase $B V R I z^{\prime}$ and $J H K$ photometry of 


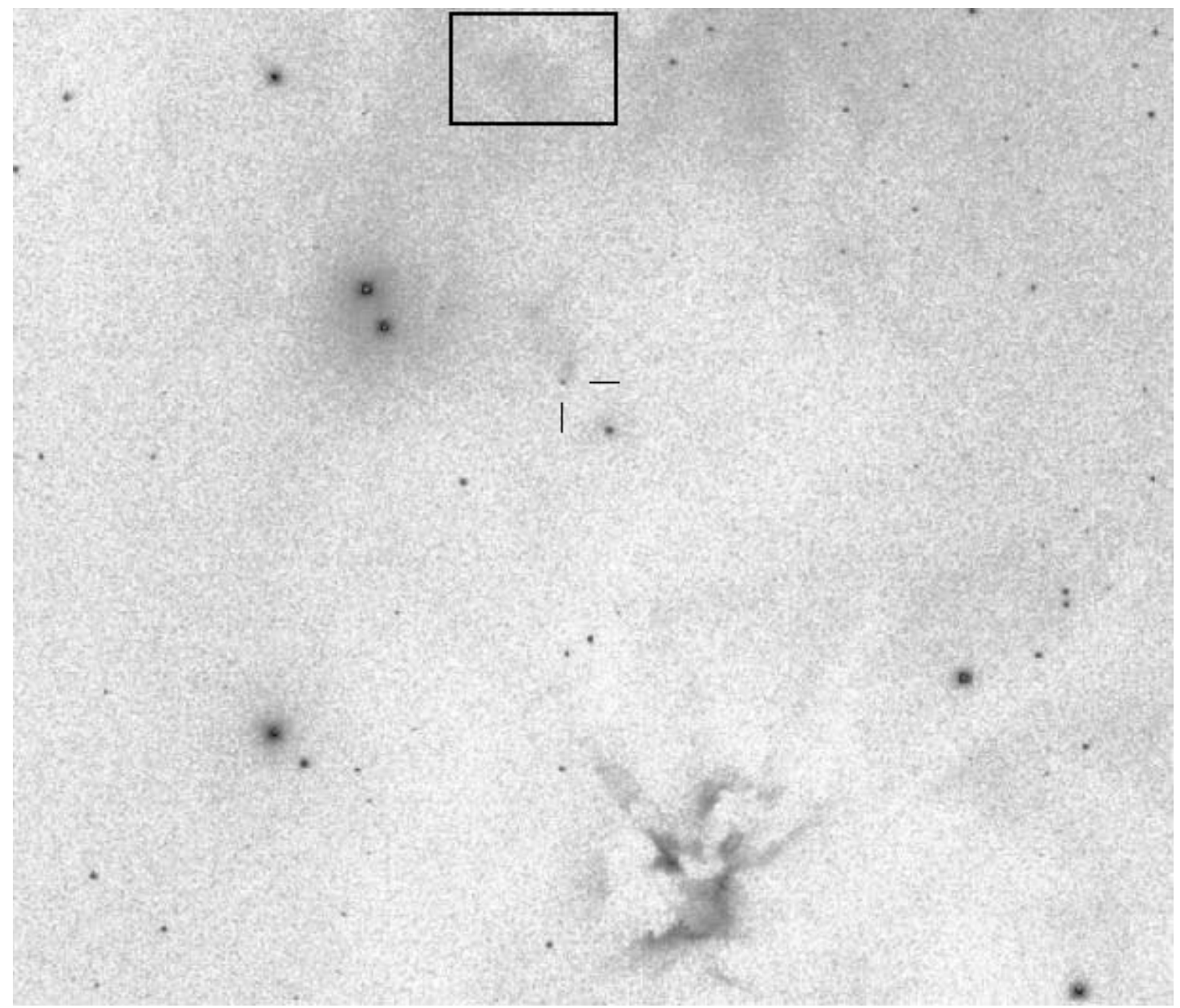

FIG. 1.-SDSS preeruption riz-band mosaic image of V1647 Ori. The location of V1647 Ori is marked on this $2 \times 2$ binned mosaic, which maps the SDSS $r, i$, and $z$ bands onto blue, green, and red, respectively. Herbig-Haro objects, such as the large HH 24 complex (bottom center), are seen in blue because of the H $\alpha$ emission appearing in the $r$ band. The image is roughly $10^{\prime}$ on a side and is displayed using a negated gray scale. North is up and east is to the left. The faint emission immediately north of V1647 Ori significantly brightens during the eruptive phase, when it is seen as McNeil's Nebula. The diffuse $r$-band emission due to HH 23, which may be driven by V1647 Ori, is highlighted by the box at top center. [See the electronic edition of the Journal for a color version of this figure.]

V1647 Ori. For $B V R I z^{\prime}$, frames were taken and flat-fielded using twilight sky flats. DAOPHOT point-spread function (PSF) fitting as implemented in IRAF was used to obtain photometric measures of the target, since there is a bright part of the nebula only a few arcseconds distant. For each data set, ensemble differential photometry was performed using a set of secondary standard stars calibrated on two photometric nights with the USNO $1.0 \mathrm{~m}$ telescope. The $z^{\prime}$ measures were relative to $z^{\prime}$ secondary standard stars calibrated by the SDSS photometric telescope (Hogg et al. 2001). For $J H K$ we used 2MASS stars in the field, eliminating obvious variables, to calibrate the data. We used a standard $K$ filter for 2004 February 11 but used a $K^{\prime}$ filter for 2004 April 12.

\section{RESULTS}

The preeruptive and recent optical and near-IR photometry of V1647 Ori are summarized in Tables 1, 2, and 3. The quiescent and eruptive-phase spectral energy distributions (SEDs) are shown as $\nu F_{\nu}$ in Figure 2.

We examine the evolution of reddening-invariant colors from the quiescent to the eruptive states to constrain the underlying physical processes. These colors take the generic form $Q_{x y z}=(x-y)-[(y-z) E(x-y) / E(y-z)]$, where $x, y$, and $z$ are the observed magnitudes in each passband. A color change is computed as $\Delta Q_{x y z}$, where color changes having $\Delta Q_{x y z}$ statistically distinct from 0 indicate changes in the SED not consistent with pure dust clearing. The conversion from $E(B-V)$ to extinction in each band follows Schlegel et al. (1998) and D. Finkbeiner et al. (2004b, in preparation).

The reddening-invariant colors measured before and during the eruption are listed in Table 4 for both $R_{V}=3.1$ and 5.5; the latter is appropriate for the larger dust grains found in star formation regions. Defined as $A_{V} / E(B-V), R_{V}$ is the ratio of the general to selective extinction.

Characteristic grain sizes are inferred to increase from 0.17 to $0.21 \mu \mathrm{m}$ as $R_{V}$ ranges from 3.1 to 5.5 , assuming a mix of silicate and carbonaceous populations (Weingartner \& Draine 2001). In the coldest portions of the molecular clouds, additional grain species such as refractory and volatile organics, olivine, water ice, orthopyroxene, trolite, and metallic iron are expected to contribute to the opacity (Pollack et al. 1994). Vacca et al. (2004) detect absorption due to water ice at $3.1 \mu \mathrm{m}$ in the near-IR outburst spectrum.

Study of the selective extinction in background stars has revealed that $R_{V}$ is not constant within the interiors of dark molecular clouds. Strafella et al. (2001) find that for the Bok globule CB $107 R_{V}$ increases inward, reaching a value of 6.5 at the core. Given the complex nature of the V1647 Ori 
TABLE 1

riz Photometry of V1647 ORI

\begin{tabular}{|c|c|c|c|c|}
\hline Date & $r$ & $i$ & $z$ & Telescope \\
\hline 1998 Nov 17 ........................ & $23.04 \pm 0.22$ & $20.81 \pm 0.05$ & $18.80 \pm 0.04$ & SDSS \\
\hline 1998 Nov $28 \ldots \ldots \ldots \ldots \ldots \ldots$ & $24.00 \pm 0.80$ & $21.03 \pm 0.09$ & $19.19 \pm 0.08$ & SDSS \\
\hline 2002 Feb $07 \ldots \ldots \ldots \ldots \ldots \ldots . . . . . . . . . .$. & $22.69 \pm 0.29$ & $20.77 \pm 0.08$ & $18.77 \pm 0.06$ & SDSS \\
\hline 2002 Feb $09 \ldots \ldots \ldots \ldots \ldots \ldots$ & $23.88 \pm 0.73$ & $21.33 \pm 0.11$ & $19.33 \pm 0.07$ & SDSS \\
\hline 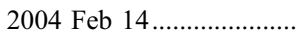 & 17.4 & 15.6 & $\ldots$ & Gemini $^{\mathrm{a}}$ \\
\hline 2004 Feb $26 \ldots \ldots \ldots \ldots \ldots \ldots$ & $\ldots$ & $\ldots$ & $14.60 \pm 0.06$ & USNO $1.55 \mathrm{~m}$ \\
\hline 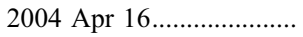 & $\ldots$ & $\ldots$ & $14.79 \pm 0.06$ & USNO $1.55 \mathrm{~m}$ \\
\hline 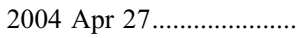 & $\ldots$ & $\ldots$ & $14.72 \pm 0.09$ & USNO $1.55 \mathrm{~m}$ \\
\hline
\end{tabular}

${ }^{\text {a }}$ From Reipurth \& Aspin (2004).

TABLE 2

Johnson-Cousins Photometry of V1647 Ori

\begin{tabular}{|c|c|c|c|c|c|}
\hline Date & $B$ & V & $R_{c}$ & $I_{c}$ & Telescope \\
\hline 2004 Feb $11 \ldots \ldots \ldots \ldots \ldots . . . .$. & & $18.52 \pm 0.07$ & $16.91 \pm 0.08$ & $14.92 \pm 0.05$ & USNO $1.0 \mathrm{~m}$ \\
\hline 2004 Feb $16 \ldots \ldots \ldots \ldots \ldots$ & $20.39 \pm 0.12$ & $18.59 \pm 0.06$ & $16.87 \pm 0.07$ & $14.90 \pm 0.04$ & USNO $1.0 \mathrm{~m}$ \\
\hline 2004 Feb $25 \ldots \ldots \ldots \ldots \ldots$ & & $18.68 \pm 0.04$ & $\ldots$ & & USNO $1.55 \mathrm{~m}$ \\
\hline 2004 Feb $26 \ldots \ldots \ldots \ldots . . .$. & $20.84 \pm 0.08$ & $18.99 \pm 0.04$ & $\ldots$ & $15.15 \pm 0.02$ & USNO $1.55 \mathrm{~m}$ \\
\hline 2004 Apr $13 . . . \ldots \ldots \ldots \ldots . . . . . . . . .$. & $20.74 \pm 0.33$ & $19.12 \pm 0.08$ & $17.24 \pm 0.09$ & $15.28 \pm 0.05$ & USNO $1.0 \mathrm{~m}$ \\
\hline 2004 Apr $16 \ldots \ldots \ldots \ldots \ldots$ & $21.13 \pm 0.07$ & $19.26 \pm 0.03$ & $\ldots$ & $15.37 \pm 0.01$ & USNO $1.55 \mathrm{~m}$ \\
\hline
\end{tabular}

TABLE 3

NeAR-IR Photometry of V1647 OrI

\begin{tabular}{|c|c|c|c|c|}
\hline Date & $J$ & $H$ & $K$ & Telescope \\
\hline 1998 Oct $07 \ldots \ldots \ldots$ & $14.74 \pm 0.03$ & $12.16 \pm 0.03$ & $10.27 \pm 0.02$ & 2MASS \\
\hline 2004 Feb 03 .......................... & $11.1 \pm 0.1$ & $9.0 \pm 0.1$ & $7.4 \pm 0.1$ & Gemini $^{\mathrm{a}}$ \\
\hline 2004 Feb 11 & $10.79 \pm 0.01$ & $8.83 \pm 0.01$ & $7.72 \pm 0.01^{\mathrm{b}}$ & USNO $1.55 \mathrm{~m}$ \\
\hline 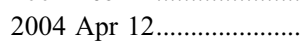 & $10.94 \pm 0.02$ & $9.06 \pm 0.02$ & $7.59 \pm 0.02^{\mathrm{b}}$ & USNO $1.55 \mathrm{~m}$ \\
\hline
\end{tabular}

${ }^{a}$ From Reipurth \& Aspin (2004).

b The USNO 1.55 m observations used a standard $K$ filter on 2004 February 11 and a $K^{\prime}$ filter on 2004 April 12, both placed on the 2MASS zero-point system but probably with transformation differences. 


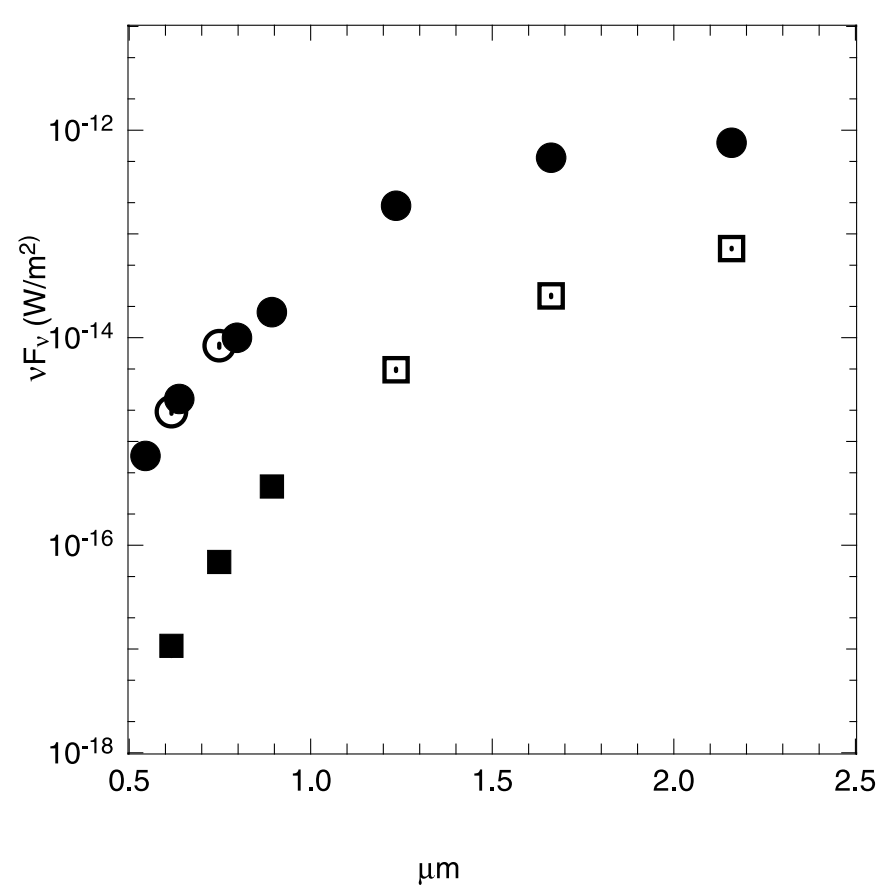

FIG. 2.-Quiescent and eruptive spectral energy distributions. The optical and near-IR SEDs are shown for the quiescent state (squares) and during eruption (circles). Data acquired by the SDSS and at the USNO are indicated by filled symbols. The open squares are the 2MASS JHK observations, and the open circles are the $r$ - and $i$-band eruptive-phase measurements of Reipurth \& Aspin (2004)

environment, including outburst cavities, accretion disks, and remnant envelopes, it is likely that $R_{V}$ can vary on small spatial scales.

\subsection{Determination of Extinction}

In the foregoing analysis we choose to characterize the intervening dust in terms of the SDSS $z$-band extinction and $R_{V}$. Since the shape of the extinction curve is dependent upon the grain size distribution for $\lambda<0.9 \mu \mathrm{m}$ (Cardelli et al. 1989), the traditional $A_{V}$ and $E(B-V)$ quantities are sensitive to both the dust column density and $R_{V}$. By adopting reference passbands longward of $0.9 \mu \mathrm{m}$ we remove the dependency on the form of the dust law, although $R_{V}$ must still be considered in the optical and UV. Alternate passbands that are free of $R_{V}$ effects include the near-IR $J$ and Johnson-Cousins $I$, the latter used by Weingartner \& Draine (2001), who adopted $A_{I} / N(\mathrm{H} \mathrm{I})=2.5 \times 10^{-22} \mathrm{~cm}^{2}$ for a standard gas-to-dust ratio.

Following Abraham et al. (2004) and Reipurth \& Aspin (2004), we assume that the quiescent-phase near-IR colors are that of an embedded low-mass classical T Tauri and obtain $E(J-H)=1.43$ mag by dereddening onto the classical T Tauri locus of Meyer et al. (1997). This value of $E(J-H)$ corresponds to $A_{J}=3.39 \mathrm{mag}\left(A_{z}=6.40 \mathrm{mag}\right)$ given $\lambda_{\mathrm{eff}}(J)=$ $1.25 \mu \mathrm{m}$ and $\lambda_{\text {eff }}(H)=1.65 \mu \mathrm{m}$ and application of the Cardelli et al. (1989) methodology.

\subsection{Changes in the Spectral Energy Distribution}

In order to analyze the general differences between the quiescent and eruptive appearance, we combine sets of observations to form representative values. The quiescent state is taken as the 1998 October 7 (2MASS $J H K$ ) and the 1998 November 17 (SDSS riz) observations. The optical photometry for the eruptive state is obtained from the 2004 February 14 Gemini $r$ and $i$ data (Reipurth \& Aspin 2004) and the 2004
TABLE 4

Reddening-Invariant Colors

\begin{tabular}{|c|c|c|}
\hline Color & $R_{V}=3.1$ & $R_{V}=5.5$ \\
\hline & $(r-i)-0.987(i-z)$ & $(r-i)-1.002(i-z)$ \\
\hline$Q_{i z J} \ldots \ldots \ldots \ldots \ldots \ldots \ldots \ldots$ & $(i-z)-0.824(z-J)$ & $(i-z)-0.605(z-J)$ \\
\hline$Q_{z J H} \ldots \ldots \ldots \ldots \ldots \ldots \ldots \ldots \ldots$ & $(z-J)-2.443(J-H)$ & $(z-J)-2.443(J-H)$ \\
\hline$Q_{\text {JHK }}$ & $(J-H)-1.563(H-K)$ & $(J-H)-1.563(H-K)$ \\
\hline
\end{tabular}

February 26 USNO $z$-band observations. The $J H K$ data for the eruptive state are from the 2004 February 11 USNO observations.

Table 5 presents the reddening-invariant colors in the quiescent and eruptive states for $R_{V}=3.1$ and 5.5. As evidenced by the $>5 \sigma$ change in all colors except for $Q_{r i z}$, which has a marginal $r$-band detection in the quiescent state, it is clear that the increase in emission is not consistent with a dust-clearing event, and thus we conclude that an intrinsic change occurred to the SED of V1647 Ori.

In Figure 3 we compare the observed magnitude variations against $\Delta A_{z}=-2$ and -4 mag dust-clearing events and, as concluded above based on reddening-invariant colors, find they are not explainable in terms of a simple diminishing of the line-of-sight extinction.

\subsection{The Quiescent Phase}

From observations of Class II protostars (classical T Tauris) we would expect that the veiling continuum due to magnetospheric accretion will be present in the SDSS $u$ and $g$ bands but weakening into the redder riz bands (Calvet \& Gullbring 1998). In a similar fashion, the thermal emission from the inner portion of the circumstellar disk should be bright in the $H$ and $K$ bands but diminishing into the bluer $J$ band (Meyer et al. 1997). For all but the most heavily veiled stars, the $i$ and $z$ bands will only be affected by extinction. None of the reddeninginvariant colors can be used to give the intrinsic spectral type.

Young (1 Myr) T Tauri stars exhibit spectral types between M0 and M4 for masses of 0.1 to $1.0 M_{\odot}$ (Baraffe et al. 1998). The use of the Meyer et al. (1997) near-IR classical T Tauri locus by Abraham et al. (2004) and Reipurth \& Aspin (2004) implicitly assumes a similar spectral type, as this locus is based on observations of $\mathrm{K} 7$ and/or M0 stars in the Taurus star formation region. The $i-z$ colors for these early to mid- $M$ spectral types range from 0.38 to 0.80 with considerable scatter on the order of several tenths of magnitudes (West et al. 2004). Given the observed $i-z(2.01 \pm 0.06)$, dereddening to these intrinsic colors requires $A_{z}=3.1-4.2 \mathrm{mag}$ for $R_{V}=3.1$ and 4.3-5.7 mag for $R_{V}=5.5$.

Dereddening using the estimated $A_{z} \sim 6.4$ and $R_{V}=3.1$ results in an intrinsic $i-z$ of -0.47 , which is too blue for the stellar locus. An assumption of $R_{V}=5.5$ yields an $i-z$ of 0.20 , corresponding to a late $\mathrm{K}$ spectral type (Finlator et al. 2000). If V1647 Ori is indeed a low-mass protostar with a late $\mathrm{K}$ or $\mathrm{M}$ spectral type, then either $R_{V}$ is high or significant $i$-band veiling is present in the quiescent state.

In Figure 4 we compare the observed quiescent-phase SED against an M0 photosphere seen under an extinction of $A_{z}=$ $6.4 \mathrm{mag}$. Excess emission is seen in the $J H K$ bands that could indicate the presence of a circumstellar disk.

\subsection{The Eruptive Phase}

The optical spectra acquired by Walter et al. (2004) during the outburst lack the $\mathrm{TiO}$ molecular absorption bands 
TABLE 5

Reddening-Invariant Color Changes

\begin{tabular}{|c|c|c|c|c|c|c|}
\hline \multirow[b]{2}{*}{ COLOR } & \multicolumn{3}{|c|}{$R_{V}=3.1$} & \multicolumn{3}{|c|}{$R_{V}=5.5$} \\
\hline & Quiescent $^{\mathrm{a}}$ & Eruptive $^{\mathrm{b}}$ & $N \sigma^{\mathrm{c}}$ & Quiescent $^{\mathrm{a}}$ & Eruptive $^{\mathrm{b}}$ & $N \sigma^{\mathrm{c}}$ \\
\hline 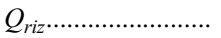 & $0.25 \pm 0.23$ & $0.81 \pm 0.10$ & 2.2 & $0.22 \pm 0.23$ & $0.80 \pm 0.11$ & 2.3 \\
\hline$Q_{i z J} \ldots \ldots \ldots \ldots \ldots \ldots \ldots \ldots \ldots \ldots \ldots \ldots$ & $-1.34 \pm 0.08$ & $-2.14 \pm 0.09$ & -6.7 & $-0.45 \pm 0.07$ & $-1.31 \pm 0.09$ & -7.7 \\
\hline$Q_{z J H} \ldots \ldots \ldots \ldots \ldots \ldots \ldots \ldots$ & $-2.24 \pm 0.12$ & $-0.98 \pm 0.07$ & 9.4 & $-2.24 \pm 0.12$ & $-0.98 \pm 0.07$ & 9.4 \\
\hline$Q_{J H K} \cdots \cdots \cdots \cdots \cdots \cdots \cdots \cdots \cdots$ & $-0.37 \pm 0.07$ & $0.23 \pm 0.03$ & 8.0 & $-0.37 \pm 0.07$ & $0.23 \pm 0.03$ & 8.0 \\
\hline
\end{tabular}

${ }^{\text {a }}$ The quiescent state is defined by the 1998 October 7 2MASS and 1998 November 17 SDSS data.

b The eruptive-phase data are from the 2004 February 14 Gemini $r$ and $i$, the 2004 February 26 USNO $z$, and the 2004 February 11 USNO JHK measurements.

${ }^{c} N \sigma$ is the ratio of color change to the quadrature sum of measurement errors.

characteristic of late $\mathrm{K}$ and $\mathrm{M}$ stars. Walter et al. (2004) attribute this to either an early photospheric spectral type or overwhelming veiling. We adopt the latter interpretation given the apparent change to the intrinsic SED and the large brightness increase in the optical (Fig. 3).

If the new component to the SED is due to an EXor outburst, then we expect to see emission from the high-temperature (6000-8000 K) inner disk (Bell et al. 1995). In Figure 5 we show the observed flux $\left(\nu F_{\nu}\right)$ increase in comparison with a $7000 \mathrm{~K}$ blackbody reddened by $A_{z}=3.2$ and $6.4 \mathrm{mag}$ for $R_{V}=3.1$ and 5.5. In common with Walter et al. (2004), we see that a single EXor-like high-temperature component can not reproduce the observed excess in both the optical and the near-IR.

For the partial dust-clearing events suggested by Reipurth \& Aspin (2004) and Walter et al. (2004) we find that, because of the increase in optical depth toward the blue, the additional flux due to a new source must fall more rapidly at the shorter wavelengths. The observed change in $J-H$ is $-0.61 \mathrm{mag}$, which, if entirely due to dust clearing, requires $\Delta A_{z}=-3.2 \mathrm{mag}$. The

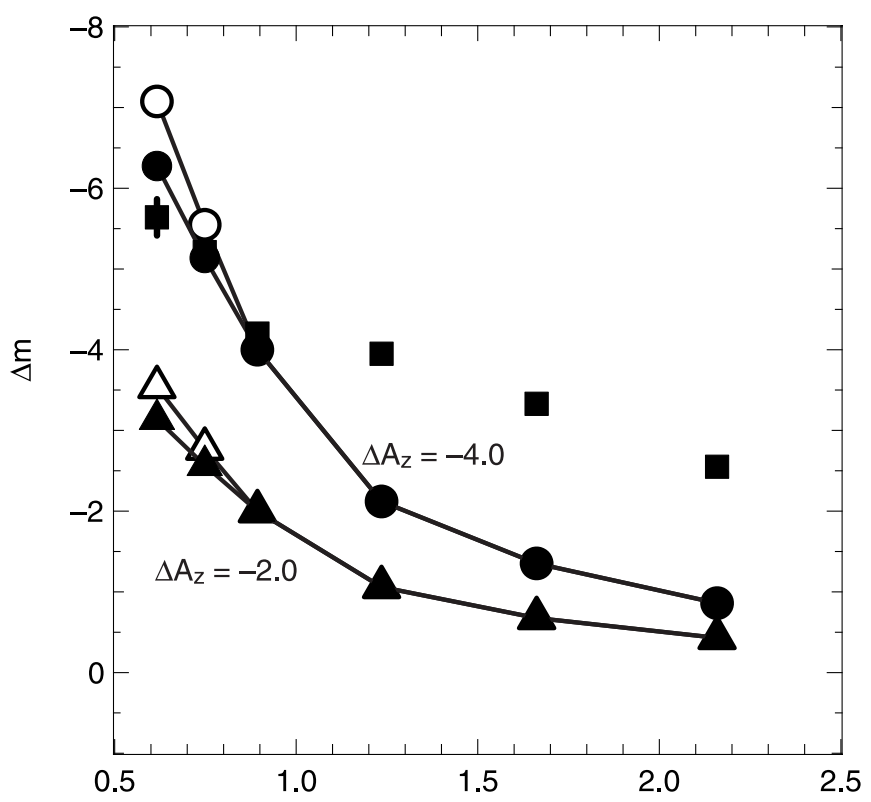

$\mu \mathrm{m}$

FIG. 3.- Changes in spectral energy distributions. The observed magnitude differences (squares) are compared against pure dust-clearing events for $\Delta A_{z}=-2 \mathrm{mag}$ (circles) and $\Delta A_{z}=-4 \mathrm{mag}$ (triangles) and for $R_{V}=3.1$ (filled) or $R_{V}=5.5$ (open) dust laws. intrinsic flux increase required in this case is shown in Figure 5 and would require either a lower temperature for the new source or a significantly higher extinction than anticipated.

Spectral indices are commonly defined by the ratio of the flux of the feature $\left(F_{S}\right)$ and that of the nearby pseudocontinuum $\left(F_{c}\right)$, so the intrinsic measure of the feature strength is $I_{0}=F_{s} / F_{c}$ (Gizis 1997). If a veiling continuum is present, defined by $F_{v}=r F_{c}$, then the measured spectral index, $I$, is

$$
I=\frac{F_{s}+r F_{c}}{F_{c}+r F_{c}}=\frac{I_{0}}{1+r}+\frac{r}{1+r} .
$$

Figure 6 shows the observed depression of the line relative to the continuum level $(1-I)$ on a logarithmic scale as a function of the veiling, $r$, for unveiled indices of $I_{0}=0.0-0.9$ in steps of 0.1. From Figure 3 we see that the flux in the $i$ band has increased by $\Delta$ mag $=-5$ or a factor of $\sim 100$, which

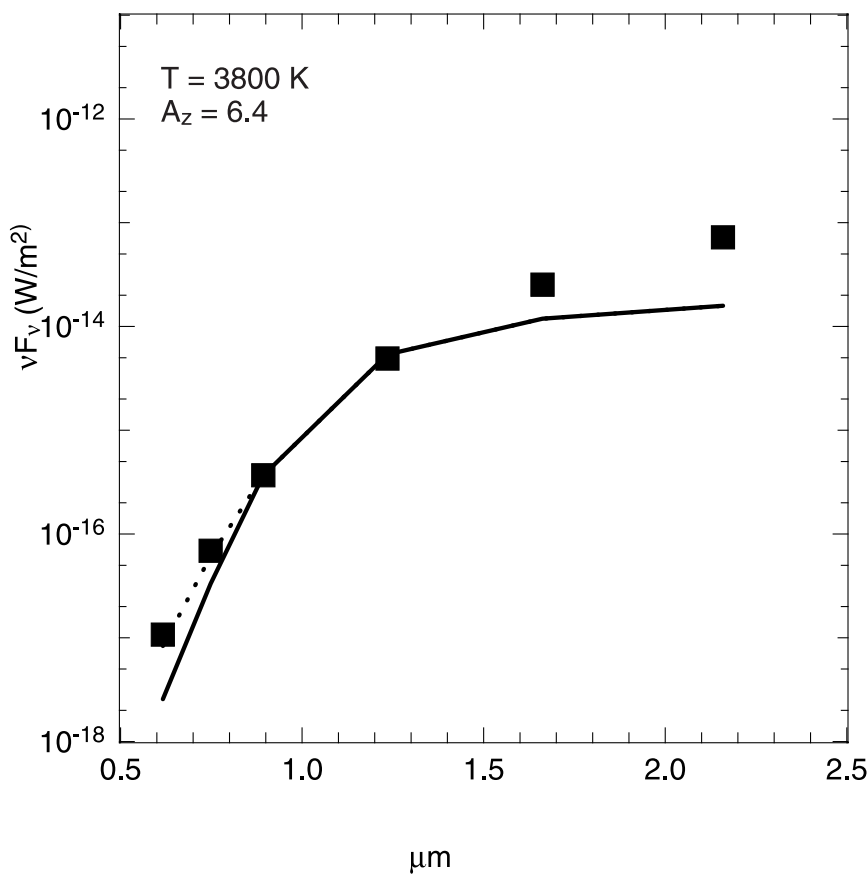

FIG. 4.-Modeling the quiescent spectral energy distribution. An observed preeruption SED is compared with an M0 photosphere $(3800 \mathrm{~K})$ seen under a line-of-sight reddening of $A_{z}=6.4 \mathrm{mag}$ for $R_{V}=3.1$ (solid line) and $R_{V}=5.5$ (dotted line). Excess emission is evident longward of $1.2 \mu \mathrm{m}(J)$. 


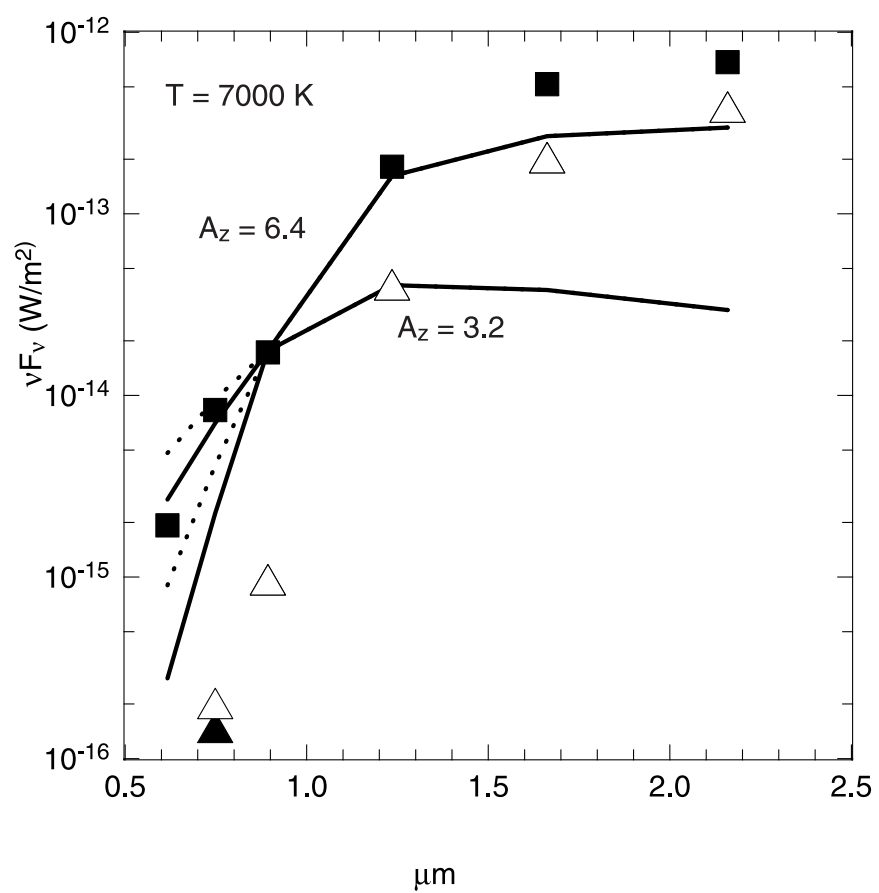

FIG. 5.-Modeling the eruptive component. This figure compares the observed flux increase ( filled squares) against a $7000 \mathrm{~K}$ blackbody viewed under $\left(R_{V}=3.1\right.$ [solid lines] and 5.5 [dotted lines]) extinctions of $A_{z}=3.2$ and 6.4 mag, scaled to match the observed change in the $z$-band flux. The triangles show the required intrinsic flux increase in the case of a $\Delta A_{z}=-3.2 \mathrm{mag}$ partial dust clearing for $R_{V}=3.1$ (filled) and 5.5 (open).

means the TiO features will be visible as a $<1 \%$ fluctuation in the observed continuum.

\section{CONCLUSIONS}

The observed photometric variations are not consistent with a simple dust-clearing event as evidenced by changes in reddeninginvariant indices. We infer that the process of eruption involves changes to both the optical and near-IR SED.

Application of an inferred preoutburst extinction of $A_{z}=$ 6.4 mag suggests a late $\mathrm{K}$ spectral type if the $r-i$ and $i-z$ colors are minimally affected by the veiling continuum arising from magnetospheric accretion shocks and if $R_{V}$ has a value expected for a star formation region. An early to mid-M spectral type is possible if the $i$ band includes an excess emission of 0.2 to $0.6 \mathrm{mag}$, corresponding to a veiling between 0.2 and 0.7 .

We interpret the quiescent phase of V1647 Ori as an embedded low-mass classical $\mathrm{T}$ Tauri. A comparison with a reddened M0 photosphere shows a near-IR excess that is presumably due to thermal emission from a normal circumstellar disk.

The outburst SED is dominated by the new component. We see that a single blackbody having the $\sim 7000 \mathrm{~K}$ temperature expected for an EXor inner disk can not simultaneously reproduce both the optical and near-IR portions of the SED. It is possible, as suggested by Reipurth \& Aspin (2004), that a partial dust-clearing event occurred in combination with an intrinsic brightening. We note that a reduction of the line-ofsight extinction would preferentially lessen the required flux increase at shorter wavelengths. This steepening of the curve shown in Figure 5 would increase the difficulty of fitting the

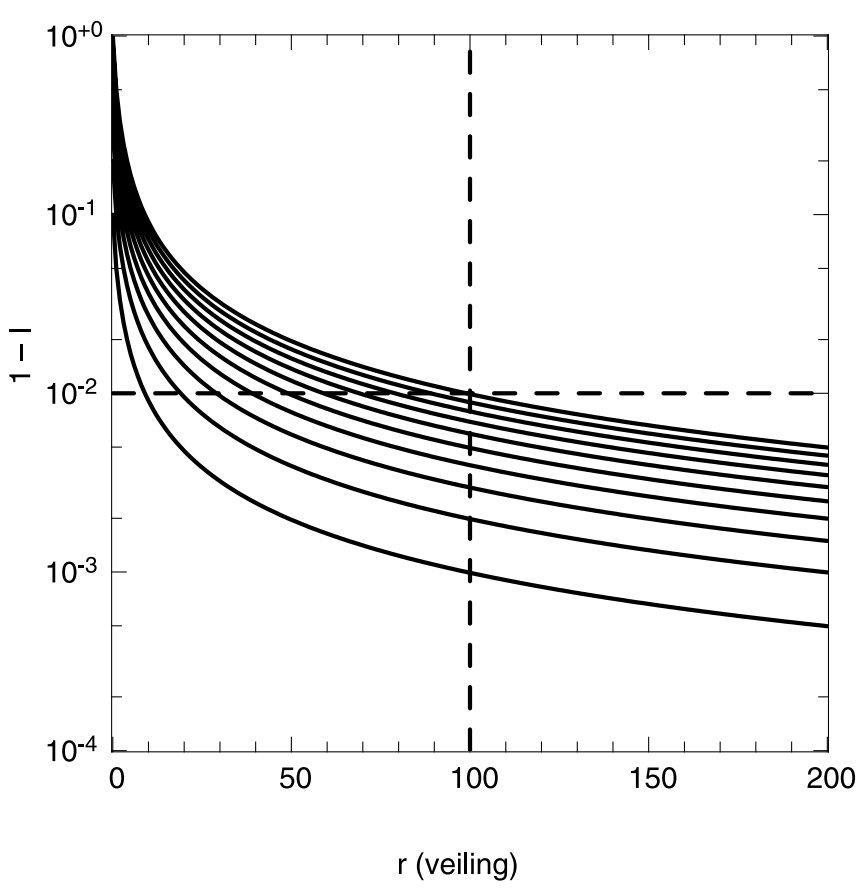

FIG. 6.-Effect of veiling on spectral features. This figure plots the observed depression of an absorption line relative to the continuum level $(1-I)$ on a logarithmic scale as a function of the veiling, $r$, for unveiled indices of $I_{0}=0.0-0.9$ in steps of 0.1 . The dashed lines indicate the eruptive-phase $i$-band veiling of 100 and a relative depression level of $1 \%$.

high-temperature (B spectral type) component observed by Briceño et al. (2004).

Further study of V1647 Ori planned using the facilities at USNO, Apache Point Observatory, and the Spitzer Space Telescope may clarify the nature and evolutionary state of this young star.

Funding for the creation and distribution of the SDSS Archive has been provided by the Alfred P. Sloan Foundation, the Participating Institutions, the National Aeronautics and Space Administration, the National Science Foundation, the US Department of Energy, the Japanese Monbukagakusho, and the Max Planck Society. The SDSS Web site is http:// www.sdss.org/. The SDSS is managed by the Astrophysical Research Consortium (ARC) for the Participating Institutions. The Participating Institutions are The University of Chicago, Fermilab, the Institute for Advanced Study, the Japan Participation Group, The Johns Hopkins University, Los Alamos National Laboratory, the Max-Planck-Institut für Astronomie (MPIA), the Max-Planck-Institut für Astrophysik (MPA), New Mexico State University, University of Pittsburgh, Princeton University, the United States Naval Observatory, and the University of Washington. This publication makes use of data products from the Two Micron All Sky Survey, which is a joint project of the University of Massachusetts and the Infrared Processing and Analysis Center/California Institute of Technology, funded by the National Aeronautics and Space Administration and the National Science Foundation. Finally, we thank the referee, Colin Aspin, for his helpful comments. 
Abazajian, K., et al. 2003, AJ, 126, 2081

Abraham, P., et al. 2004, A\&A, 419, L39

Andrews, S. M., Rothberg, B., \& Simon, T. 2004, ApJ, 610, 45

Anthony-Twarog, B. J. 1982, AJ, 87, 1213

Baraffe, I., et al. 1998, A\&A, 337, 403

Bell, K. R., et al. 1995, ApJ, 444, 376

Briceño, C., et al. 2004, ApJ, 606, 123

Calvet, N., \& Gullbring, E. 1998, ApJ, 509, 802

Cardelli, J. A., Clayton, G. C., \& Mathis, J. S. 1989, ApJ, 345, 245

Clark, F. O. 1991, ApJS, 75, 611

Eislöffel, J., \& Mundt, R. 1997, AJ, 114, 280

Finkbeiner, D., et al. 2004a, AJ, in press

Finlator, K., et al. 2000, AJ, 120, 2615

Fukugita, M., Ichikawa, T., Gunn, J. E., Doi, M., Shimasaku, K., \& Schneider, D. P. 1996, AJ, 111, 1748

Gizis, J. E. 1997, AJ, 113, 806

Gunn, J. E., et al. 1998, AJ, 116, 3040

Herbig, G. H. 1977, ApJ, 217, 693

Herbig, G. H., et al. 2001, PASP, 113, 1547

Hodapp, K.-W., et al. 1996, ApJ, 468, 861
REFERENCES

Hogg, D. W., Finkbeiner, D. P., Schlegel, D. J., \& Gunn, J. E. 2001, AJ, 122,2129

Lis, D. C., Menten, K. M., \& Zylka, R. 1999, ApJ, 527, 856

McNeil, J. W. 2004, IAU Circ., 8284, 1

Meyer, M. R., Calvet, N., \& Hillenbrand, L. A. 1997, AJ, 114, 288

Pier, J. R., Munn, J. A., Hindsley, R. B., Hennessy, G. S., Kent, S. M., Lupton, R. H., \& Ivezić, Ž. 2003, AJ, 125, 1559

Pollack, J. B., Hollenbach, D., Beckwith, S., Simonelli, D. P., Roush, T., \& Fong, W. 1994, ApJ, 421, 615

Reipurth, B., \& Aspin, C. 2004, ApJ, 606, 119

Schlegel, D., Finkbeiner, D., \& Davis, M. 1998, ApJ, 500, 525

Smith, J. A., et al. 2002, AJ, 123, 2121

Stoughton, C., et al. 2002, AJ, 123, 485

Strafella, F., et al. 2001, ApJ, 558, 717

Vacca, W. D., et al. 2004, ApJ, 609, 29

Walter, F. M., Stringfellow, G. S., Sherry, W. H., \& Field-Pollatou, A. 2004, AJ, 128,1872

Weingartner, J. C., \& Draine, B. T. 2001, ApJ, 548, 296

West, A., et al. 2004, AJ, 128, 426

York, D., et al. 2000, AJ, 120, 1579 